\section{Im Möbelwagen auf dem Königsweg zum Unbewussten}

Hanspeter Mathys (Zürich)

Zusammenfassung: Die Deutung der Träume gilt seit Freud als Königsweg zur Kenntnis des Unbewussten. Erstaunlich klar beschreibt er in seinen Texten, wie die Analytiker über das sorgfältige Hören aufdie Assoziationen ihrer Analysanden den latenten Bedeutungsgehalt der mitgeteilten Träume deuten können. Für Fritz Morgenthaler war dieser Zugang über die Assoziationen zu bewusstseinsnah. Er postulierte eine Traumdiagnostik, bei der es darum geht, die Traumtendenz anhand dessen, wie die Analysandin mit ihrem Traum umgeht, zu ermitteln. Erst dann könne und solle man sich mit den Assoziationen des Träumers befassen. Gemäss dieser Auffassung werden Träume nicht einfach mitgeteilt sondern erzählend agiert. Dieser für die Traumanalyse hoch relevante Ansatz wird in diesem Text theoretisch weiter entwickelt und anhand einer Fallvignette diskutiert. Dabei interessiert vor allem die Frage, wie Analytiker durch das Agieren der Traummitteilung in ein Enactment verwickelt werden und was dies für die Traumanalyse bedeutet.

Schlüsselwörter: Traumanalyse, Traumdiagnostik, Funktion von Traummitteilungen, Containment, Enactment

\section{Der Traum als «vollkommen asoziales Produkt»}

Der Traum ist ein vollkommen asoziales seelisches Produkt; er hat einem anderen nichts mitzuteilen; innerhalb einer Person als Kompromiss der in ihr ringenden seelischen Kräfte entstanden, bleibt er dieser Person selbst unverständlich und ist darum für eine andere völlig uninteressant. Nicht nur dass er keinen Wert auf Verständlichkeit zu legen braucht, er muss sich sogar hüten, verstanden zu werden, da er sonst zerstört würde, er kann nur in der Vermummung bestehen. (Freud, 1905, S. 204)

Dass Freud die Interpretation der Träume als Königsweg zum Verständnis des Unbewussten bezeichnete, ist hinlänglich bekannt. Dass er den Vorgang der Traummitteilung in der analytischen Situation so skeptisch sah, ist aufs Engste mit seiner Traumtheorie verflochten. Träume sind primär nicht dazu gedacht, erinnert, erzählt und schon gar nicht verstanden zu werden. Das würde ihre Funktion gefährden: Der Traum ist in erster Linie der Hüter des Schlafs, so Freud. Damit er diese Funktion erfüllen kann, darf er gar nicht allzu klar sein. Denn wäre er dies, würde sich das Ich empört in aller Form distanzieren oder verängstigt Alarm schlagen, damit der Träumer sofort aufwache. Ein Vorgang, den Fritz Morgenthaler sehr anschaulich mit seiner Bühnenmetapher erläutert hat (Morgenthaler, 1986, S. 84). Das nächtliche Aufwachen und Erinnern eines Traums ist eine Panne des psychischen Systems. Neuere Erkenntnisse aus der empirischen Traumforschung verdeutlichen dies: Die durchschnittliche Traumdauer pro Nacht betrage etwa drei Stunden, sagen uns die Traumforscher. Das Aufwachen und Erinnern bildet dabei die Ausnahme (Leuschner, 1999; Ermann, 2005). Mit anderen Worten: die Traumarbeit erledigt ihren Job, den Schlaf zu schützen, recht ordentlich.

Die Hintergründe, warum Freud die Traumitteilungen seiner Patienten dann eben doch sehr willkommen waren, sind allgemein bekannt: Bei seinen Bemühungen, den seltsam vermummten Traumgebilden auf die Spur zu kommen, entdeckte Freud hinter dem erinnerbaren von ihm so genannten manifesten Traum, latente Traumgedanken, die aber nie in ihrer ursprünglichen Form ins Bewusstsein gelangten. Erinnerbar und erzählbar ist immer nur der entstellte, manifeste Traum. Den zuständigen Mechanismus dieser Umwandlung bezeichnete er als Traumarbeit. Das Ziel seiner Traumanalyse bestand nun darin, den Weg der Traumarbeit quasi in umgekehrter Richtung zu gehen, das heisst die latenten Traumgedanken aus dem manifesten Traumtext zu rekonstruieren. Der Weg dazu ist derjenige der freien Assoziation. Der Traum wird Stück für Stück zerlegt, dem Träumer vorgelegt mit der Aufforderung, freie Einfälle dazu zu äussern. Nach und nach wird der Traum somit angereichert mit Material aus dem Leben des Träumers und in einen Kontext gestellt, aus dem heraus deutende Schlüsse gewagt werden können. Ziel dieses deutenden Prozesses ist die Ermittlung des den Traum verursachenden infantilen Wunsches, der (so Freud bis 1933) die Rolle des Kapitalisten beim Unternehmen Traumproduktion innehabe.

Dieser Umgang mit Träumen stellt eine entscheidende Wende in der Geschichte der Traumdeutung dar.

Die strikte Unterscheidung von manifestem und latentem Traum war für Freud und die Psychoanalyse viele Jahre sicherlich notwendig, um eine wissenschaftliche Traumforschung paradigmatisch zu 
begründen und sie gegenüber allen traditionellen und populärpsy-

chologischen Deutungskünsten zu schützen. (Mertens, 2000, S. 52)

Die Konsequenz dieses Vorgehens war eine Relativierung und explizite Geringschätzung des manifesten Traumtextes in seiner vorliegenden Gestalt als Traumbericht. Der Traum war für ihn vielmehr ein Bilderrätsel als ein Erzählformat. Aufgrund dieses Verständnisses war für Freud die kommunikative Handlung des Erzählens von Träumen relativ uninteressant. Dieses von ihm verächtlich als «Flickenteppich» bezeichnete Produkt der manifesten Traumerzählung war ein sekundär bearbeiteter Brocken, der vom bewussten Ich geformt wurde. Diesen Brocken von seinen manifesten Schlacken zu befreien und auf das pure Gold der latenten und damit unbewussten Traumgedanken zu stossen, darum ging es ihm letztlich.

\section{Träume werden nicht erzählt, sie werden agiert}

Für Morgenthaler war dieser Zugang über die Assoziationen zu bewusstseinsnah. Dahinter steckt die Vorstellung einer radikalen Trennung zwischen den Ebenen des Bewussten und des Unbewussten:Was bewusst sei, so Morgenthaler könne nicht gleichzeitig unbewusst sein. Nie sei das Nächstliegende das, was der Traum sagen wolle. Programmatisch zu Tage geförderte Assoziationen unterliegen exakt derselben Abwehrleistung wie die Traumarbeit bei der Produktion des manifesten Traums. Für den latenten Gehalt sei damit also noch nicht viel gewonnen. Erforderlich sei so etwas wie eine Traumdiagnostik, respektive die Ermittlung der Tendenz oder der Richtung, wo der Traum hinführe. Diese lasse sich aus dem spezifischen Umgang des Träumers mit seinem Traum ermitteln, also nur im Kontext der analytischen Situation. Erst dann, wenn sozusagen die Richtung klar ist, kann und soll man sich mit den durch Assoziationen angereicherten Inhalten befassen. Morgenthaler illustriert dies anhand einer Möbelwagen-Metapher:

Wenn wir an einen Traum herangehen, so ist es fast so, als hätten wir einen beladenen Möbelwagen auf der Strasse stehen, und suchten eine Wohnung, in die wir diese Möbel hineinstellen können. Ich sage, es ist gut, wenn man die Wohnung hat, wo man die Möbel auslädt. Die Möbel im Möbelwagen sind die Inhalte des Traumes. Die Wohnung, das ist die Tendenz, die in der Dynamik dieses Traumes zunächst aufgefunden werden muss. Wir müssen zunächst wissen, in welche Richtung diese Bedürfnisse gehen, die wir als den unbewussten Wunsch bezeichnen. Die Tendenzen in diesem Traum müssen zuerst klargestellt werden, bevor wir die Inhalte hineinlegen können. (Morgenthaler, 1986, S. 155)

Bei der Traumdiagnostik geht es um die sorgfältige Beobachtung der Begleitumstände bei der Traummitteilung. Zur Erarbeitung einer Traumdiagnostik im Morgenthaler'schen Sinn gilt es, den Blick auf formale und strukturelle Gesichtspunkte zu richten (vgl. Binswanger, 2016). Dieses Interesse für die genauen Umstände, wann, wem und wie ein Traum erzählt wird, mit anderen Worten also für den Kontext in der analytischen Situation hängt mit einer bemerkenswerten Idee zusammen: Traum und Traummitteilung sind für Morgenthaler nicht eine Form des Erinnerns von Vergessenem und Verdrängtem. Vielmehr wird durch die Tat des Träumens und des Traumerzählens das, was der Traum an Vergessenem und Verdrängtem enthält, agiert (Morgenthaler, 1986, S. 51). Das komme daher so Morgenthaler, dass Träume mit wichtigen Erlebnissen in Verbindung stünden, die in die frühe Kindheit fallen und damals ohne Verständnis erlebt worden seien Morgenthaler bezieht sich dabei auf eine Passage bei Freud:

Für eine besondere Art von überaus wichtigen Erlebnissen, die in sehr frühe Zeiten der Kindheit fallen und seinerzeit ohne Verständnis erlebt worden sind, nachträglich aber Verständnis und Deutung gefunden haben, lässt sich eine Erinnerung meist nicht erwecken. Man gelangt durch Träume zu ihrer Kenntnis ... Immerhin erfordert dieser Gegenstand soviel kritische Vorsicht und bringt soviel Neues und Befremdendes, dass ich ihn einer gesonderten Behandlung an geeignetem Material vorbehalte. (Freud, 1914, S. 129)

Morgenthaler weist darauf hin, dass Freud diese in Aussicht gestellte Arbeit nie realisiert habe. Nach seiner Auffassung

wäre das geeignete Material, an dem diese Betrachtung weitergeführt werden könnte, der Traum, seine Erzählung und der Umgang des Träumers mit ihm, sofern wir neben der inhaltsbezogenen Traumdeutung traumdiagnostische Betrachtungen anstellen und unsere besondere Aufmerksamkeit auf die Funktion des Traumgeschehens richten. (Morgenthaler, 1986, S. 52) 
Morgenthaler hat dies in zahlreichen Traumseminaren auf der Ebene der ganz konkreten Arbeit mit Träumen aufgezeigt, aber weniger in der Theorie.

\section{Agieren statt symbolisieren}

Die Idee, dass im kommunikativen Geschehen einer Traummitteilung mehr geschieht als nur ein Austausch von Worten, eben eine Handlung, eine Interaktion, die unerhört verdichtetes Material beinhaltet, von dem der Träumer nicht weiss, was es mit ihm zu tun hat, wirft die Frage auf, woraus denn dieses Traummaterial besteht. Freud war bis 1933 überzeugt davon, dass es regelmässig ein verdrängter, infantilerWunsch sei, der die Traumbildung in Gang setze. Als auslösendes Moment und als Material für die Gestaltung der Traumszenerie bediene sich das Traum-Ich dann zweitens des aktuellen Tagesgeschehens, das als Tagesrest in den Traum einfliesse. Damit fliessen zwei auch zeitlich sehr unterschiedliche Quellen im Traum zusammen. Mit der Revision der Traumlehre (Freud, 1933) unter dem Eindruck kriegstraumatisierter Patienten ergänzte er seine Traumtheorie dahingehend, dass auch erlebte Traumata, dem Paradigma des Wiederholungszwangs gehorchend, in das Traumgeschehen Eingang finden können. Und wenn Morgenthaler (und Freud) davon ausgehen, dass Träume mit wichtigen Erlebnissen in Verbindung stehen, die in die frühe Kindheit fallen und damals ohne Verständnis erlebt wurden, scheint die Bandbreite an möglichen Trauminhalten schier unendlich zu sein. Neben infantilen Wünschen könnten dies beispielsweise eben auch traumatisierende Kindheitserlebnisse sein, die ohne Verständnis erlebt wurden und nie symbolisiert werden konnten. Dies wären dann keine verdrängten Wünsche oder Erinnerungen, die im Rahmen der Traumanalyse bewusst gemacht werden können. Es handelt sich um eine andere Kategorie von Träumen, entsprechend ist auch eine andere technische Haltung dazu erforderlich.

An diesem Punkt sind neuere Ansätze zur Traumtheorie und zur Technik der Traumanalyse entstanden. Diese Erweiterung der Traumtheorie geht einher mit einer Weiterentwicklung der psychoanalytischen Behandlungstechnik überhaupt, die erforderlich wurde, weil sich im Laufe der Jahrzehnte auch Patienten mit einer schwereren strukturellen Beeinträchtigung in psychoanalytische Behandlung begaben, als dies bei Freuds Klientel der Fall war. Unter zahlreichen neueren Darstellungen (vgl. die Übersicht bei Deserno, 2007, S. 918) sei die Arbeit von Weiss (2002) herausgegriffen. Weiss bezieht sich auf Bion (1962), der davon ausging dass Traumerfahrungen den Beginn psychischen Lebens darstellten. Bion hat sich vorwiegend mit Traumanalysen bei Borderline- und psychotischen Patienten beschäftigt, was von Meltzer (1984) und Segal (1991) weiter systematisiert und in
Zusammenhang gebracht wurde mit dem Konzept der Symbolisierung und der Störung der Traumbildung. Rohde-Dachser (1983) hat diesen Ansatz ausformuliert und mit wichtigen Hinweisen zur modifizierten Technik der Traumanalyse angereichert. Der wichtigste Unterschied zur Freudschen Traumtheorie besteh darin, dass sich die Symbolisierungsstörung auf die Traumbildung so auswirkt, dass die Fähigkeit zur Traumarbeit fehlt oder nur sehr eingeschränkt zurVerfügung steht, dass also eine Umformung der latenten Traumgedanken in einen manifesten Traum nicht stattfinden kann, und damit die Bedingung für die Schlaf schützende Funktion des Traums wegfällt. ${ }^{1}$

Ausgehend nun von diesem Ansatz entwirft Weiss ein Koordinatensystem welches auf der einen Achse die Struktur, auf der zweiten Achse den Gebrauch des Traummaterials in der analytischen Situation erfasst. Weiss versteht diese Achsen als Kontinuum. Auf der einen Seite wird ein Traum erzählt mit dem Ziel, über die innere Welt zu kommunizieren. Auf der anderen Seite geht es um Evakuierung von unverdaulichem Material, das nicht in der eigenen Psyche verarbeitet werden, sondern nur in ein Gegenüber mittels projektiver Identifikation evakuiert werden kann (Weiss, 2002, S. 635). Dabei geht es um einen Versuch, den Analytiker mit einem Teil des Selbst des Patienten oder mit einem seiner inneren Objekte zu identifizieren und so in ein Enactment hineinzuziehen. Dabei ist entscheidend, dass der Analytiker in der Lage ist, zu erkennen, wie er in die interne Welt des Patienten involviert ist. Indem er dies formuliert, vermag er eine dritte Position zu etablieren, von der aus er den Weg aus dem Enactment herausfinden kann. Dabei geht Weiss davon aus, dass der Evakuierungs- oder auf Analytikerseite der Containment-Aspekt vor allem bei solchen Patienten mit einer Symbolisierungsstörung, die sich bei der Traumbildung auswirkt, von Bedeutung ist.

Im Zusammenhang mit der oben beschriebenen Idee Morgenthalers, dass Träume nicht erzählt, sondern agiert werden, stellt sich nun die Frage, ob die eben skizzierte Funktion von Traummitteilungen nur bei Patienten mit einer Symbolisierungsstörung relevant ist oder ob der Traum nicht an sich entwicklungspsychologisch gesehen ein sehr frühes psychisches Gebilde ist, das ganz generell eher agiert als erzählt wird. In diesem letzteren Sinne interpretiert jedenfalls Deserno (2007) das Modell von Meltzer (1984). Meltzer könne, so Deserno, mithilfe des Bionschen «container-contained»-Modells Voraussetzungen des Träumens konzeptualisieren, die entwicklungspsychologisch vor Freuds Konzept des Primärvorgangs lägen. 
Primär körperliche Bedürfnisspannungen werden in frühe Symbolformen oder Protosymbole transformiert. Dann erst erhalten sie durch den Primärvorgang ihre bildhaft-symbolische und durch den Sekundärvorgang ihre sprachsymbolische Ausdrucksgestalt. (Deserno, 2007, S. 918)

Und Mertens meint, der Ausgangspunkt (für solche ohneVerständnis erlebten Eindrücke aus der Kindheit) sind Interaktionserfahrungen und Beziehungseindrücke der frühen Lebenszeit, die jenseits der Sprache und eines elaborierten Symbolverständnisses und zeitlich noch vor einem entwickelten deklarativen, autobiografischen Gedächtnis entstanden sind.

Diese frühen sinnlichen Interaktionsformen bilden das eigentlich Unbewusste, nicht nur das psychodynamische Verdrängte, sondern dasjenige Unbewusste, wohin die Sprache nicht reicht, und das doch ständig nach bildlicher und sprachlicher Symbolisierung verlangt. (Mertens, 2005/2006, S. 43 f.)

Zusammenfassend lässt sich ein kommunikatives Agieren dadurch kennzeichnen, dass es sich um präsymbolische Mitteilungsformate handelt. Dies kann dann der Fall sein, wenn die psychische Fähigkeit zur Symbolisierung nicht ausreichend entwickelt werden konnte oder aber, und dies ist der Ansatzpunkt von Morgenthaler, wenn es sich um Material handelt, das sehr frühe Erfahrungen beinhaltet, die ohne Verständnis und damit ohne Symbolisierungsmöglichkeit erlebt wurden. Die damit verbundene sehr grundsätzliche Frage, was eigentlich das Unbewusste ausmacht, soll hier nicht weiterverfolgt werden. Vielmehr interessiert die Frage, was es für die traumanalytische Arbeit bedeutet, wenn mittels eines Traumes so kommuniziert wird, dass man eher von einer Handlung als einer verbalen Mitteilung ausgehen sollte.

\section{Konsequenzen für die Traumanalyse}

Die Rollenzuweisungen beim Unterfangen «Traumanalyse» folgten bei Freud einem relativ klaren Skript nach folgendem Schema: Traummitteilung Assoziationen - Deutung (s. oben). Die Zielsetzung war ebenfalls klar: ergründen, welche unbewussten infantilen Wünsche der Traum beinhaltet. Das Ernstnehmen der Aussage, dass Träume nicht einfach nur erzählt, sondern agiert werden, erfordert eine Ausweitung der Fragestellung: nicht nur, was bedeutet ein Trauminhalt, sondern vielmehr, was wird eigentlich mit der Handlung der Traummitteilung zum Ausdruck gebracht? Wie wird ein Traum eingesetzt und welche latente Funktion hat dessen Mitteilung in diesem konkreten Moment der analytischen Situation? Und was bedeutet dies ganz praktisch für die Haltung des Analytikers als Gegenüber im Moment der Traumitteilung? Moser (2003) macht darauf aufmerksam, dass die Ausgangslage, wie mit Träumen in der Analyse umzugehen sei, keineswegs für beide Beteiligten die gleiche ist. Er beschreibt die analytische Situation als einen Ort, an dem zwei unterschiedliche Traumtheorien aufeinandertreffen. Sie müssten aneinander justiert werden, wenn die Interpretation verstanden werden soll. Es ist davon auszugehen, dass die potenziellen Differenzen der impliziten Traumtheorien zwischen Analysand und Analytiker erst mit der Zeit deutlicher werden, wenn überhaupt. Was wäre nun, wenn diese Frage nach der jeweils unterschiedlichen Traumtheorie gar nie zur Sprache käme und bis zum Schluss der Analyse zwei Traumtheorien quasi wie zwei parallel laufende Filme nebeneinander herliefen?

Statt weiteren theoretischen Ausführungen möchte ich im folgenden Abschnitt versuchen, anhand einer Fallvignette diese Frage zu erläutern. Damit ist auch der Versuch verbunden, die oben erwähnte Idee Morgenthalers, wonach Träume unverstandene Erlebnisse enthalten, konsequent fortzuführen und weiter zu entwickeln. Auch die oben aufgeworfene Frage, ob das Container-Modell und damit zusammenhängende Enactment-Phänomene nur bei Patienten mit einer Symbolisierungsstörung eine Rolle spielen oder ob sie die Traum-Kommunikation generell bestimmen, soll anhand des klinischen Materials vertieft werden.

\section{Eine Fallvignette: Amalie X}

Der folgende Abschnitt zeigt, wie eine Analysandin und ein Analytiker über einen Traum sprechen. Die Analyse dieser Fallvignette basiert auf einer umfang reichen Einzelfallstudie (vgl. Mathys, 2010). Datengrundlage dieser Einzelfalluntersuchung ist die psychoanalytische Psychotherapie einer Patientin mit dem Pseudonym Amalie X (Kächele et al., 2006). Von der 531 Stunden umfassenden Behandlung wurden 517 Stunden per Tonband aufgezeichnet. Die interessierende Passage ereignet sich in Stunde 224.

Diese Stunde beginnt damit, dass die Analysandin Amalie erzählt, sie hätte einen Autounfall gehabt und dabei das eigenartige Gefühl bekommen, sie hätte diesen Unfall aktiv gewollt: «Es isch blöd, aber je länger ich darüber nachdenk, wie das wirklich war, ist wirklich einer in mich reingekahren bin.» Durch diese eigenartige Formulierung, bei der Subjekt und Verbform nicht zusammenpassen, entsteht eine Verwirrung, wer den Unfall eigentlich verursacht hat. Im weiteren Verlauf 
macht Amalie deutlich, was sie damit meint, wenn sie sagt, sie habe den Unfal gewollt. An einer Stelle sagt sie, der andere am Unfall Beteiligte «dringt da ein». Mit dieserWortwahl bringt sie zum Ausdruck, dass sich dieser Unfall wie eine sexuelle Penetration angefühlt habe. Damit macht sie ihrem Analytiker deutlich, worüber sie eigentlich sprechen möchte: nicht über die Fakten des Unfallhergangs, sondern über ihr subjektives Gefühl, ihre (sexuelle) Fantasie, die sie damit verbindet. Dabei wird das Auftreten dieser Vorstellung sowohl zeitlich als auch inhaltlich ausgeweitet. Nicht erst anlässlich dieses Unfalls sei der Wunsch nach einem Auffahrunfall entstanden, sondern schon vorher. An einer Stelle wird dieser Wunsch ins Aktive gewendet: «wie schön wär das wenn man da jetzt mal auf jemand drauffahren könnte.» Während die passiven Wendungen dieser Wunschfantasie, dass eben ein anderer in sie hineinfahren möge aufgrund ihrer eigenen Wortwahl bereits als sexuell konnotierte Fantasie beschrieben wurde, ändert sich mit der Wendung vom Passiven ins Aktive auch die inhaltliche Tönung der Fantasie: jetzt als Aktive fände sie es schön, auf jemand anderen drauffahren zu können und dabei etwas kaputt zu machen. Gleichzeitig verwirrt sie diese Vorstellung. Dies hängt aber nicht in erster Linie mit den sexuellen Fantasien zusammen, sondern mit der Frage, wer wem aktiv hineinfährt. Dies zeigt sich nochmals in folgender Formulierung: "das kam aber alles erst hinterher, dringt in jem- mich, in jemand mich, ach pfui!» Der Analytiker fragt nach: «Bezog sich das «pfui nicht auch auf einen Gedanken der sich gerade so mit eingeschlichen hatte, dass SIE eindringen oder?» Amalie reagiert sofort darauf: «Weil ich mich versprochen hab, gell. Mir ist aufgefallen, dass ich mich versprochen hab, aber ich kann jetzt nix sagen, weil ich hab umgekehrte Gedanken gehabt.» Stattdessen fällt ihr eine Fantasie aus ihrer Klosterzeit ein, wo sie von Pfarrern und Ministranten in ihren langen Gewändern umgeben war, durch die sie in ihrer Fantasie hindurchsieht. Und kurz danach berichtet sie folgenden Traum:

Ich hab da noch ganz eigenartig weitergeträumt, in der Nacht darauf, dass ich mit dem Auto fuhr und wurde auch vorne angefahren von einer Frau, und der habe ich dann eine Krippen-oder eine Puppen-, ich glaube eine Puppenstube weggenommen. Es war eine ganz alte Frau und das war auch so unklar, wer schuld ist in meiner Vorstellung. Und ich fuhr dann weiter und wurde dann von rechts hinten angefahren, und dann noch von vorne. Das Auto war dann ziemlich also im vorderen wirklich kaputt und plötzlich stand ich in der Mitte und ein ganzer Kreis von Autofahrern stand um mich rum, eine ganze Menge Männer, es war ein richtiger Kreis und ich hab denen dann ganz ruhig und ganz genau formuliert die Bedingungen, zweitens das drittens das und hab aber immer das Gefühl gehabt, ich muss ganz schnell noch die Puppenstube zurückbringen. Und am Schluss hab ich dann noch die Bedingung formuliert, das weiss ich noch genau, und nun müssen sie eine absolute Abtretungserklärung an mich auch unterschreiben. Und da scholl ein schallendes Gelächter mir entgegen, und ich bin dann aufgewacht, ziemlich heftig daran aufgewacht.

Der Analytiker kommentiert diesen Traum im Zusammenhang des bisherigen Stundenverlaufs. Insbesondere die Formulierung im Zusammenhang mit dem Einfall zu den Klerikern, die durch die langen Gewänder hinten und vorne gleich seien, und die (hier nicht wieder gegebene) Erwartung Amalies, dass der Analytiker aufgrund ihrer Fantasien erschrocken sei, dienen ihm als Kontext zum Verstehen des Traums. Diese Formulierung «vorn und hinten gleich» respektive im Anschluss an diesen Traum «vorn und hinten kaputtgemacht» führt er mehrmals ins Gespräch ein, und sie dient ihm zu folgender Deutung, die hier in paraphrasierter Form etwa so lautet: Sie erwarten, dass ich erschrecke, weil diese Forderung, dass Männer alles abtreten, was sie haben, auch an mich gerichtet ist. Wenn wir davon reden, was die Männer haben - im Unterschied zu den Frauen - dann reden wir vom männlichen Geschlechtsteil. Es geht also darum, dass die Männer und auch ich meinen Penis abtreten sollen. Das ist der eigentliche Grund, warum Sie meinen, ich sollte erschrecken.

Dies sei der Kern der Abtretensforderung. Amalie wolle, dass die Männer im Traum und der Analytiker ihr das, was sie als männlich auszeichnet, übergeben. Der Traum zeige, so der Analytiker weiter, Amalie als eine beschädigte Frau - beschädigt von einer alten Frau übrigens - die vorne kaputtgemacht wurde, so dass ihr nun dort etwas fehlt. Sie sieht sich nun berechtigt, an die Männerwelt die juristisch einwandfrei begründete Forderung zu stellen, dass diese ihr zurückerstatten, was ihr kaputtgemacht wurde. Im Traum geht diese Forderung maximal schief: Sie wird von den anwesenden Männern ausgelacht. Die Forderung ist jedoch nicht nur an die Männer im Traum gerichtet, sondern auch an den Analytiker-Mann im Hier und Jetzt. Mit der Mitteilung des Traums wird diese Forderung nun implizit an ihn adressiert.

Diese Abtretensforderung an den Analytiker-Mann als Wiedergutmachung erlittener Beschädigung bestimmt in Stunde 224 noch in weiteren Varianten die 
Interaktion. In einem der folgenden Redebeiträge spitzt der Analytiker seine Deutung nochmals zu, wenn er eine Gesprächspassage mit folgenden Worten kommentiert: “Ja vielleicht haben Sie mich in dem Moment zum Priester gemacht.» $\mathrm{Zu}$ verstehen ist diese Äusserung immer noch auf dem Hintergrund des Bildes von den langen Gewändern der Priester, die sie so aussehen lassen, als wären sie «vorne und hinten gleich», also geschlechtslos. In der Tat entgegnet Amalie auf diese Äusserung dann, dass sie es als durchaus angenehm empfindet, wenn der Analytiker «im Priestergewand auftrete», eben als geschlechtsloses Wesen. Diesem «beruhigenden» Gefühl, wie Amalie es formuliert, stellt der Analytiker entgegen, dass ihr dann aber auch etwas Wichtiges fehlen würde, nämlich sein Erschrecken über die Abtretensforderung. Denn, ganz simpel formuliert: wo nichts ist, kann nichts abgetreten werden und kann auch kein Erschrecken über entsprechende Abtretensforderungen stattfinden.

Ganz zum Schluss dieser sehr dichten Passagen wird diese Abtretensforderung an den Analytiker auf bemerkenswerte Weise konkretisiert:

Amalie: «...das ist das, was ich schon oft empfunden hab und auch schon mal gesagt hab, dass die Analytiker nicht alles sagen ...dass sie eben sehr viel zurückhalten ...dann ist mein Verdacht, dass Sie als weiser Mann und als Priester und als Guru da hinten sitzen und vieles zurückhalten, was mir dann fehlt.»

Analytiker: «hm, und was was äh, habe Sie da beson-woran denken Sie da besonders jetzt auch im ..."

Amalie (fällt ihm ins Wort): « ...an Interpretation denke ich.»

Im Gespräch über den Traum mit der Abtretungsforderung kommt nun eine weitere Dimension ins Spiel. Was ihr fehlt, ist das, was der Analytiker ihr vorenthält, weil er nicht alles sagt, was er denkt und weiss. Sie konkretisiert dies, indem sie das, was ihr dann fehlt, benennt: es handelt sich um Interpretation. Damit ermöglicht diese Passage eine Konkretisierung dessen, was im Anschluss an den Traum als Abtretungsforderung an die Männer gerichtet war. Hier und jetzt an den einen Mann in der Analyse tritt sie mit der Forderung heran, dass dieser ihr die Kunst der Interpretation abtritt. Offensichtlich nimmt Amalie diese als phallische Qualität des Analytikers wahr. Im Zusammenhang mit der oben dargestellten Dynamik des eigenen Beschädigtseins ist diese Abtretungsforderung an den Analytiker als Entschädigung und Wiedergutmachung zu verstehen. Sie verlangt seine Interpretations-Potenz. Am liebsten so, dass er sie nicht mehr hat, was in einem symbolischen Verständnis hiesse, dass sie ihn seiner phallischen Potenz berauben, ihn kastrieren würde. Was in Stunde 224 angedeutet erscheint, wird sich in den folgenden Stunden der Analyse verdeutlichen: Dieser offensichtliche Kastrationswunsch hat für Amalie dann doch auch etwas Unheimliches und mobilisiert Angst. ${ }^{3}$ Diese unverhohlene Kastrationslust, die entsprechende Ängste auf den Plan ruft, mündet in einen Kompromiss, dessen Entwicklung etwa folgendermassen beschrieben werden kann:

1) Männer haben etwas, was Amalie fehlt.

2) Sie will das auch haben. Diesen Wunsch nach männlicher Ausstattung wehrt sie ab.

3) Weil sie das, was die Männer haben, nicht haben kann, sollen wenigstens die Männer das auch nicht haben (Kastrationswunsch): Männer und Frauen sollen vorne gleich sein; es soll keine Geschlechterdifferenz geben.

4) Das bezieht sich auch auf den Analytiker.

5) Aber auch dieser Wunsch geht zu weit. Beide Wünsche müssen abgewehrt werden: als Frau einen Penis zu wollen oder ihn den Männern wegzunehmen.

6) Eine Abtretensforderung fungiert als Kompromiss: von den Männern wird Entschädigung verlangt; Amalie will Anteil haben an dem, was sie haben.

7) Bezogen auf den Analytiker heisst das: Partizipation an seinen phallischen Qualitäten.

8) Besonders attraktiv erscheint ihr dessen Fähigkeit zur (Traum-)Interpretation.

\section{Enactment: Verborgene Wege der Wunscherfüllung}

Die in der Analyse der Stunde 224 formulierten Befunde sind nicht bloss eine Momentaufnahme, sondern zeigen eine Entwicklung, die sich bis zum Analyse-Ende in vielen der folgenden Traumstunden nachzeichnen lässt und als Makromuster der analytischen Beziehung zwischen Amalie und ihrem Analytiker etwa folgendermassen skizziert werden kann:

1) Amalie geht es nicht um eine dialogisch-kooperativ angelegte Interpretation ihrer Trauminhalte, sondern um den Akt des Traum-Interpretierens an sich.

2) Ihr Ziel ist es, die Kunst der Traum-Interpretation, der sie phallische Qualität beimisst, zu erlernen und zu beherrschen.

3) Der Weg dazu führt über die Partizipation an der als phallisch-männlich wahrgenommenen Kunst der Interpretation des Analytikers.

Der Analytiker wird von Amalie nicht als dialogischer Partner behandelt, mit dem sie in kooperativer Art und Weise die latente Bedeutung ihrer Träume erforscht. 
Vielmehr verstrickt sie ihren Analytiker in ein phallisches Rivalisieren darum, wem letztlich die Traumdeutungs-Hoheit zukommt. Es handelt sich dabei allerdings um eine durchaus zweischneidige und als solche konflikthafte unbewusste «Strategie» Amalies: einerseits soll der Analytiker gute Trauminterpretationen bieten, damit sie daran partizipieren kann. Anderseits wird der Analytiker regelrecht kastriert hinsichtlich seiner Deutungs-Potenz. Das zeigt sich in der distanzierten Haltung bis zur expliziten Ablehnung seiner Deutungen. Das Zweischneidig-Konflikthafte besteht in einer an sich nicht zu vereinbarenden Positionierung des Analytikers: einerseits soll er Experte sein, an dessen Interpretationskunst sie partizipieren will, anderseits ist er Rivale, der das Nachsehen haben soll. Der wunschgemässe Ausgang aus dieser Konstellation sieht konsequenterweise so aus, dass sie als «Lehr-Analysandin» ihren Lehrer überflügelt, so dass sie als Frau bezüglich Trauminterpretations-Kompetenz letztlich besser phallisch ausgestattet ist als der männliche Analytiker. In einem letzten Traum (Stunde 517) auf die Frage des Analytikers, was ihr diese Analyse gebracht habe, stellt sie diesen Wunsch insofern als erfüllt dar, als sie diejenige ist, von der man als Expertin wissen will, was Interpretation ist.

Wie geht nun der Analytiker mit dieser Inszenierung um? Es spricht vieles dafür, dass er sich in dieses Enactment hineinziehen lässt. Der zentrale Konflikt Amalies kreist um die beschriebenen Themen der Geschlechterdifferenz und Kastration und kann nicht psychoanalytisch bearbeitet werden. Gerade dadurch wird er prägend für die interpersonelle Dynamik. Mit anderen Worten: dieser Teil wird nicht bearbeitet sondern agiert. So scheint es zu folgendem unbewussten Pakt zu kommen: Der Analytiker deutet die Träume Amalies inhaltlich, auch mit Bezug zu den konflikthaften Themen. Damit stösst er zwar oft auf Widerstand, was den Inhalt betrifft, gleichzeitig erfüllt er genau durch diese Handlung der Trauminterpretation den Partizipationswunsch Amalies. Er agiert mit, indem er inhaltlich Deutung an Deutung liefert. Somit erlangt sie eine Teil-Befriedigung oder Genugtuung ihres Restitutionswunsches statt einer Bewusstmachung und einem Durcharbeiten des zentralen Konflikts. Damit ist der Analytiker nicht mehr (idealtypischer) Ko-Konstrukteur der Traumanalyse, sondern Ko-Akteur in einem unbewussten Pakt zwischen ihm und seiner Analysandin.

Diese spezifische Situation hat Streeck (1998) überzeugend herausgearbeitet. Die Pointe seines Ansatzes liegt darin, dass sich unbewusste Wünsche interaktive Mechanismen von sozialer Ordnung zunutze machen, um ans Ziel zu gelangen, auch und gerade in der analytischen Situation. Der Analytiker dagegen will in der Regel nicht, dass der Patient seine Wünsche in der Analyse befriedigt. Vielmehr soll er sich dieser Wünsche mithilfe der Deutungen des Analytikers bewusst werden.
Angewandt auf die Funktion der Traumkommunikation Amalies besteht der entscheidende Punkt darin, dass diese Deutungen des Analytikers den Gegenstand der Wunscherfüllung bei Amalie bilden. Sie bedient sich des in der Psychoanalyse höchst willkommenen Mitteilungsformats der Traumschilderung, weicht dann aber insofern davon ab, als sie sich nicht wie eine idealtypische Rezipientin der Traumdeutungen ihres Analytikers verhält. Vielmehr verändert sich ihre Rolle zusehends vom Subjekt ihrer Träume hin zur Ko-Deuterin und -Forscherin des Phänomens Traum an sich. Sie entfernt sich damit vom Patientinnen-Status hin zur valablen Konkurrentin des Analytikers auf dem Feld der Traumdeutung. Wunscherfüllung geschieht hier also nicht in ganz offensichtlicher Abweichung von der zu erwartenden interaktiven Ordnung beim Traumdialog, sondern in deren Vollzug. Gerade diese in psychoanalytischen Sitzungen etablierte Ordnung, dieses interaktive Prinzip des Traumdialogs dient Amalie dazu, den Wunsch nach Reparatur ihrer als beschädigt empfundenen Weiblichkeit als erfüllt zu betrachten. ${ }^{4}$

Nach Streeck entfalten solche Gesten eine zwingende Kraft und veranlassen das Gegenüber zu einem ganz bestimmten Verhalten oder drängen es zur Übernahme einer bestimmten Rolle. Er beschreibt dies als Mitagieren des Analytikers. Gleichzeitig verweist er damit den Anspruch einer neutralen Haltung des Analytikers in das Reich der Illusionen. Welche Kraft dem unbewussten Wunsch innewohnt, zeigt sich also einerseits an dem suggestiven Druck, der auf den Analytiker ausgeübt wird, aber auch an der Reaktion der Patientin, wenn die etablierte Interaktionsordnung von Seiten des Analytikers «unbeabsichtigt durchbrochen wird und die Patientin sich daraufhin erzürnt zurückzieht» (Streeck, 1998, S. 56). Bei Amalie findet sich eine sehr eindrückliche Passage dazu in Stunde 449. Dort berichtet sie von einem Traum ihres Partners, und sie will vom Analytiker hören, was er dazu respektive zu ihrer Deutung des Traums sagt. Er sagt aber nichts dazu, was Amalie dazu bringt, ihn «am liebsten erschiessen zu wollen».

Ganz allgemein, so Streeck, habe sich die Auffassung durchgesetzt, dass psychoanalytische Therapie ein interaktives Geschehen ist, das vom Patienten und Analytiker gemeinsam hervorgebracht wird. Somit handelt es sich bei diesem Geschehen immer um Ko-Produktionen und nicht nur um Ko-Konstruktion. Nicht selten ist der Analytiker unbewusst in sie verstrickt und erkennt dies erst, «wenn der Pausenvorhang gefallen ist» (Streeck, 1998, S. 53). Der Analytiker kann zwar nicht nicht mitagieren, er sollte jedoch im Nachhinein realisieren, in welche Szene er mit hinein verwickelt wird und welche Rolle er übernommen hat und dies dann in Form einer szenischen Deutung wiederum für die Analyse verwenden. In den untersuchten Traumstunden ist dies allerdings kaum je der Fall. 
Bei der nachträglichen Betrachtung dieser Interaktion im analytischen Prozess im Zusammenhang mit Traummitteilungen stellt sich also die Frage, warum der Analytiker die von Amalie geschilderten Träume eigentlich immer gleich behandelt, nämlich als auf der inhaltlichen Ebene zu interpretierende Erzählsequenzen. ${ }^{5}$ Es fragt sich, warum er so gut wie nie eine Bemerkung auf der Metaebene macht, was mit der und durch die Traummitteilung interaktiv geschieht, respektive was Amalie durch die Traummitteilung und den anschliessenden Traumdialog mit ihm «macht». Warum sagt er nie so etwas wie «Sie haben gerade fünf Träume in einer Stunde erzählt, da können wir ja gar nicht alles besprechen». Oder: «Sie erzählen mir vermehrt Träume von anderen Personen und gar nicht mehr Ihre eigenen, was könnte das heissen?» Oder: «In letzter Zeit habe ich den Eindruck, dass Sie an meinen Beiträgen zum Traum nicht mehr sonderlich interessiert sind. Wie kommt das?» Er scheint nie dieses bestimmte Interaktionsmuster zu deuten, diese Inszenierung Amalies als Rivalin um die Deutungshoheit und «stille Teilhaberin» an seinen Interpretationen. Mit anderen Worten, er macht so gut wie nie eine szenische Deutung des im Zusammenhang mit der Traumschilderung sich etablierenden interaktiven Plots. Somit trägt der Analytiker seinen Teil dazu bei, dass die Analyse sich mehr und mehr zu einer Weiterbildungsveranstaltung für Traum-Interpretation entwickelt.

Der bemerkenswerte Verzicht auf eine solche szenische oder Übertragungsdeutung zu diesem interaktiven Muster im Umgang mit den Traummitteilungen dürfte mit der Sonderstellung der Traummitteilung im Rahmen psychoanalytischer Behandlungssettings zusammenhängen. Es gibt eine ausführliche Debatte um diese Sonderstellung des Traums (vgl. Greenson, 1970; Ermann, 1998). Bei aller Diskrepanz ist doch davon auszugehen, dass Träume in den psychoanalytischen Behandlungszimmern in der Regel willkommene Gäste sind. Oft ist in der psychoanalytischen Literatur auch die Rede davon, dass die Mitteilung eines Traums ein Geschenk an den Analytiker darstelle (Pontalis, 1974; Morgenthaler, 1986; Ermann, 1998). Dies ist auch beim Analytiker Amalies der Fall. Mehr noch verschiedene Passagen legen den Schluss nahe, dass es ihm ganz gelegen kommt, über das triangulierende Moment des Traums zu kommunizieren. Es gibt Stellen, wo Amalie direkt und sehr unvermittelt die Beziehung zum Analytiker anspricht, er jedoch eher ausweicht. Auf diesem Hintergrund erscheint der Traum für den Analytiker in der Tat als ein Geschenk, der aber gerade dadurch sein verführendes Potenzial entfaltet und damit den analytischen Blick auf die damit verbundene Inszenierung zu trüben vermag. Erst diese Vorzugsstellung des Traums ermöglicht diese spezifische Form des Enactment.

\section{Fazit}

Mit dem Blick auf den dialogischen Umgang mit dem Traum verschiebt sich die Perspektive weg vom Trauminhalt hin auf die Funktion der Traummitteilung Es ist dies, ein konversationsanalytischer Zugang mit dem Versuch einer psychoanalytischen Deutung des Geschehens und damit der Versuch einer Analyse, die von der Oberfläche des sprachlichen Geschehens ausgeht, das Manifeste ernst nimmt und von da aus versucht, das Latente zu verstehen.

Ich fürchte, Freud würde sich im Grabe umdrehen bei dieser Vorgehensweise. Und Morgenthaler? Die dargestellte Analyse wäre beiden wahrscheinlich zu bewusstseinsnah. Beiden war es ein zentrales Anliegen, bei der Traumanalyse von einer strikten Trennung zwischen Bewusstem und Unbewusstem auszugehen. Die für Freud zentralen Analyseschritte, die Mechanismen der Traumarbeit zu bestimmen, werden hier so gut wie gar nicht berücksichtigt. Hingegen wurde die Entdeckung Morgenthalers, dass Träume erzählend agiert werden, in dieser Arbeit aufgegriffen und mit dem Konzept des Enactments konsequent weiter gedacht. Dabei wurde allerdings darauf verzichtet, einen Zusammenhang zwischen formalen Gesichtspunkten und dem Trauminhalt eines ganz konkreten Traums herzustellen, wie dies bei den Traumseminaren Morgenthalers geschah. ${ }^{6}$ Für die hier dargestellte Sichtweise schliesse ich mich der Einschätzung von Mertens an, die etwas mehr Freiheitsgrade in der Bestimmung des Zusammenhangs von Form und Inhalt des Traums ermöglicht: «Wir alle kennen Patienten, bei denen die Traumerzählung ein Ausagieren der unbewussten Konfliktthematik, die konkordant mit dem unbewussten Trauminhalt, aber auch unabhängig davon sein kann, darstellt.» (2005/2006, S. 40). Diese Sicht plädiert dafür, den Trauminhalt und die Funktion des Traumberichts unabhängig voneinander zu verstehen und in der jeweiligen Situation immer wieder neu zu bestimmen. Als Analytikerinnen und Analytiker sind wir, ganz mit Morgenthaler gesprochen, gefordert, eine Traumdiagnostik vorzunehmen; das heisst, Traumschilderungen sowohl von ihrem Inhalt her als auch auf ihre Funktion hin in der analytischen Situation zu betrachten. Je nachdem steht das eine oder das andere im Vordergrund. Je nachdem verdeckt das eine das andere. Je nachdem ergänzen sich beide Perspektiven. Das Berücksichtigen der formalen Gesichtspunkte, oder in einem hier dargestellten umfassenderen Verständnis, der Funktion der Traummitteilung, eröffnet damit eine mögliche Nebenstrasse zum besseren Verstehen des Unbewussten, neben dem Königsweg. 


\section{Literatur}

Binswanger, M. (2016). Dream Diagnostics: Fritz Morgenthaler's Work on Dreams.

The Psychoanalytic Quarterly 85, 727-757.

Bion, W. R. (1962). Learning from experience. London: Heinemann.

Deserno, H. (2007). Traumdeutung in der gegenwärtigen psychoanalytischen

Therapie. Psyche-Zeitschrift für Psychoanalyse und ihre Anwendungen 61, 913-942.

Ermann, M. (2005). Träume und Träumen. Hundert Jahre «Traumdeutung». Stuttgart: Kohlhammer.

Ermann, M. (1998). Träume erzählen und die Übertragung. Forum der Psychoanalyse 14, 95-110.

Freud, S. (1933). Revision der Traumlehre. In Gesammelte Werke, Bd. 15, (S. 6-31). Frankfurt a. M.: Fischer.

Freud, S. (1914). Erinnern, Wiederholen, Durcharbeiten. In Gesammelte Werke, Bd. 10, (S. 126-136). Frankfurt a. M.: Fischer.

Freud, S. (1905). Der Witz und seine Beziehung zum Unbewussten. Gesammelte Werke, Band 6. Frankfurt a. M.: Fischer.

Freud, S. (1900). Die Traumdeutung. Gesammelte Werke, Band 2. Frankfurt a. M. Fischer.

Greenson, R. R. (1970). Die Sonderstellung des Traums in der psychoanalytischen Praxis. In H. Deserno (Hrsg.), DasJahrhundert der Traumdeutung (S. 140-161). Stuttgart: Klett-Cotta.

Kächele, H., Albani, C., Buchheim, A., Grünzig, H.-J., Hölzer, M., Hohage, R., Jimenez, J. P., Leuzinger-Bohleber, M., Mergenthaler, E., Neudert-Dreyer L., Pokorny, D. \& Thomä, H. (2006). Psychoanalytische Einzelfallforschung: Ein deutscher Musterfall Amalie X. Psyche - Zeitschrift für Psychoanalyse und ihre Anwendungen 60, 387-425.

Leuschner, W. (1999). Experimentelle psychoanalytische Traumforschung. In H. Deserno (Hrsg.), Das Jahrhundert der Traumdeutung (S. 356-374). Stuttgart: Klett-Cotta.

Leuzinger-Bohleber, M. (1989). Veränderung kognitiver Prozesse in Psychoanalysen. Band 2. Fünf aggregierte Einzelfallstudien. Berlin, Heidelberg, New York, London, Paris, Tokyo: Springer.

Mathys, H. (2010). Wozu werden Träume erzählt? Interaktive und kommunikative Funktionen von Traummitteilungen in der psychoanalytischen Therapie. Giessen: Psychosozial.
Meltzer, D. \& Theusner-Stampa, G. (1984). Traumleben. Eine Überprüfung der psychoanalytischen Theorie und Technik. München usw.: Verlag Internationale Psychoanalyse.

Mertens, W. (2005/2006). Anmerkungen zu Fritz Morgenthalers Buch «Der Traum». Journal für Psychoanalyse 45/46, 31-51.

Mertens, W. (2000). Einführung in die psychoanalytische Therapie. (3., überarbeitete Auflage). Stuttgart: Kohlhammer.

Morgenthaler, F. (1986). Der Traum. Fragmente zur Theorie und Technik der Traumdeutung. Frankfurt: Campus.

Moser, U. (2003). Traumtheorien und Traumkultur in der psychoanalytischen Praxis (Teil 1). Psyche - Zeitschrift für Psychoanalyse und ihre Anwendungen 57, 639-657.

Müller-Pozzi, H. (2008). Eine Triebtheorie für unsere Zeit. Sexualität und Konflikt in der Psychoanalyse. Bern: Huber.

Pontalis, J.-B. (1974). Der Traum als Objekt. In H. Deserno (Hrsg.), Das Jahrhundert der Traumdeutung (S. 205-223). Stuttgart: Klett-Cotta.

Rohde-Dachser, C. (1983). Träume in der Behandlung von Patienten mit schweren Ichstörungen. In M. Ermann (Hrsg.). Der Traum in Psychoanalyse und analytischer Psychotherapie (S. 107-119). Berlin, Heidelberg, New York: Springer. Segal, H. (1991). Dream, Phantasy and Art. London: Tavistock.

Streeck, U. (1998). Verborgene Wege der Wunscherfüllung. In B. Boothe (Hrsg.), Über das Wünschen (S. 48-66). Göttingen: Vandenhoeck \& Ruprecht.

Weiss, H. (2002). Reporting a Dream Accompanying an Enactment in the Transference Situation. International Journal of Psycho-Analysis 83, 633-645.

\section{Anmerkungen}

Wer einmal erlebt hat, wie quälend Patienten mit einer Borderline-Organisation das nächtliche Zu-Bett-Gehen, das Einschlafen und das nicht symbolisierte (Alb-)Träumen erleben, kann sich eineVorstellung davon machen, was für ein Privileg eine gut funktionierende Traumarbeit für den Schlaf darstellt.

Die Gesprächspassagen wurden der besseren Lesbarkeit wegen sprachlich geglättet. Zum konversationsanalytischen Originaltranskript und zur detaillierten Analyse vgl. Mathys, 2010, S. $141 \mathrm{ff}$

3 Die psychoanalytischen Konzepte «Penisneid» und «Kastrationskomplex» sind gleichermassen zentral wie auch umstritten. Entscheidend ist, dass es sich nicht um objektive Konzepte handelt, die eine ontologische Geschlechterdifferenz so festlegen, sondern um eine «Theorie der imaginären und neurotischen, von den Fantasmen der frühkindlichen Sexualforschung bestimmten Verarbeitung der Kastrationsproblematik» (Müller-Pozzi, 2008, S. 152).

Wie subtil sich dieser «verborgene Weg der Wunscherfüllung» ereignet, zeigt ein Blick in die reichhaltige Forschungsliteratur zu diesem Fall Amalie X. So attestiert Leuzinger- 
Bohleber (1989) in einer Untersuchung von aggregierten Einzelfallstudien Amalie im Vergleich mit anderen AnalysandInnen die ausgeprägteste Fähigkeit zur Selbstreflexion. Ausserdem verfüge sie «in den Endstunden über ein ausgeprägtes Wissen zur Informationsaufnahme und -verarbeitung bezüglich ihrer Träume, was ihr unter anderem die Möglichkeit schafft, ihre Träume nun weitgehend selbst zu verstehen und zu deuten» (S. 77). Diese an sich nachvollziehbare Einschätzung wird hier anders interpretiert. Der eigentlich durchaus willkommene Zuwachs an eigener analytischer Traumdeutungskompetenz erfolgt bei Amalie in einem ganz bestimmten Kontext, und zwar im Dienste der Abwehr ihres zentralen Konflikts.

$5 \quad$ Eine nachträgliche Analyse von klinischem Material birgt immer die Gefahr, aus der Position des Besserwissers kritische Fragen aufzuwerfen, weil man selber nicht in das hochkomplexe Übertragungs- und Gegenübertragungsgeschehen involviert ist. Der Analytiker von Amalie X hat sich ganz bewusst diesem Risiko ausgesetzt, weil er die klinisch-psychoanalytische Forschung vorantreiben wollte. Die Wertschätzung gegenüber der Bereitschaft von Analytiker und Analysandin, diesen intimen Prozess des analytischen Geschehens der Forschung zugänglich zu machen, überwiegt die hier geäusserte kritische Analyse bei weitem.

$6 \quad$ Ich verdanke R. Binswanger den präzisierenden Hinweis, wie Morgenthaler diesen Zusammenhang sah: Der formale Umgang mit Traumerzählungen erlaube Rückschlüsse auf die Dynamik von Widerstand und Übertragung sowie auf die Mechanismen der Zensur durch das unbewusste Ich und lege dadurch erst das Verständnis des Trauminhalts frei. Insofern könne man von einer widersprüchlichen Einheit von Trauminhalt und formalen Aspekten der Traummitteilung sprechen, jedoch nicht im Sinne eines unmittelbaren Rückschlusses vom Formalen auf den Inhalt.

\section{Angaben zum Autor}

Hanspeter Mathys, Studium der Klinischen Psychologie und Psychopathologie an der Universität Zürich, Psychoanalytische Weiterbildung am Psychoanalytischen Seminar Zürich (PSZ), Klinische Lehr- und Wanderjahre in Münsterlingen und Männedorf. Seit 2009 in eigener psychoanalytischer Praxis. 2009 Promotion an der Universität Zürich zum Thema der kommunikativen und interaktiven Funktionen von Traummitteilungen in der psychoanalytischen Therapie. Seit 2014 Leiter der Psychologischen Beratung für Studierende an der Pädagogischen Hochschule Zürich (PHZH). 